\title{
A Estabilidade do Estado Homogêneo em Metapopulações com Estrutura Etária
}

M.L. de CASTRO ${ }^{1}$, J.A.L. da SILVA², Departamento de Matemática Pura e Aplicada, Universidade Federal do Rio Grande do Sul, Av. Bento Gonalves, 9500, Porto Alegre, RS, Brasil.

\begin{abstract}
Resumo Neste trabalho obtemos um modelo discreto para uma metapopulação com estrutura etária. Mostramos que um mecanismo de migração dependente somente da idade dos indivíduos não pode vir a estabilizar o ponto de equilíbrio homogêneo de um sistema previamente instável na ausência de migração, mas pode tornar instável um conjunto desacoplado de populações estáveis, o que caracteriza a migração como um processo desestabilizador.
\end{abstract}

\section{Introdução}

Vários trabalhos destacam o quanto o efeito da migração sobre a estabilidade do estado homogêneo em metapopulações pode ser importante, devendo ser considerado na construção de um modelo matemático que descreva a evolução de uma espécie distribuída em fragmentos onde ocorra migração (ou dispersão) [5, 6]. Assim como a estrutura espacial, a estrutura etária de uma população é importante, principalmente quando estamos interessados na evolução de uma população sobre um período comparável ao tempo de vida dos indivíduos [7]. Além disso, muitas vezes o movimento migratório está fortemente relacionado com a idade dos indivíduos.

O primeiro passo na construção de modelos que incorporam estrutura etária e espacial foi dado por Hastings [2], que propôs um modelo para uma população com duas classes etárias e analisou os efeitos do movimento migratório com taxas de migração específicas para cada classe em uma rede de 2 patches. Neste trabalho estendemos o modelo sugerido por Hastings para uma população estruturada em $N$ classes etárias, distribuída em uma rede de $n$ sítios. Nosso objetivo é analisar o efeito da migração dependente da idade dos indivíduos em uma metapopulação com estrutura etária. A seguir, introduzimos o modelo local utilizado, o que nos servirá de base para analisar os efeitos causados somente pela dispersão.

\footnotetext{
1 manuela@mat.ufrgs.br

${ }^{2}$ jaqx@mat.ufrgs.br
} 


\section{Modelo Local}

O modelo local considera dois processos: reprodução e sobrevivência. Para o processo de reprodução, vamos representar por $f_{i}$ a taxa de fertilidade dos indivíduos na classe $i$ e para o processo de sobrevivência, vamos denotar por $p_{i}$ a taxa de sobrevivência dos indivíduos da classe $i, i=1,2, \ldots, N$. Em populações naturais estes parâmetros podem depender de vários fatores, mas aqui vamos considerá-los constantes. Considerando intervalos de tempo e idade iguais e denotando por $x_{t}^{i} \mathrm{o}$ número de indivíduos na classe etária $i$ no tempo $t$, obtemos o sistema

$$
\mathbf{x}_{t+1}=L \mathbf{x}_{t},
$$

onde $\mathbf{x}=\left(x^{1}, \ldots, x^{N}\right)^{T}$ e $L$ é a Matriz de Leslie [7],

$$
L=\left[\begin{array}{ccccc}
f_{1} & f_{2} & \cdots & f_{N-1} & f_{N} \\
p_{1} & & & & \\
& p_{2} & & & \\
& & \ddots & & \\
& & & p_{N-1} &
\end{array}\right]
$$

Alguns parâmetros importantes para o sistema (2.1) devem ser definidos. Definimos $l_{i}$ como a probabilidade de um indivíduo nascido alcançar a classe etária $i$,

$$
l_{i}=\prod_{j=1}^{i-1} p_{j} \quad i=2, \ldots, N .
$$

Note que $l_{1}=1$, pois $l_{1}$ representa a probabilidade de um indivíduo nascido alcançar a classe etária 1. Assim, o número reprodutivo básico da população é dado por

$$
R_{0}=\sum_{i=1}^{N} f_{i} l_{i}
$$

A partir dos parâmetros $l_{i}, i=1 \ldots, N$ e $R_{0}$, podemos obter a distribuição etária da fertilidade, definindo

$$
m_{i}=\frac{l_{i} f_{i}}{R_{0}}, \quad i=1, \ldots, N
$$

Note que $\sum_{i=1}^{N} m_{i}=1$, pois $m_{i}$ representa o percentual de contribuição da classe etária $i$ para a estimativa $R_{0}$.

Além destas $N$ classes, vamos considerar a existência de uma classe inferior $w$, onde se encontram os recém gerados. Dependendo da espécie em questão, esta classe pode ser vista como a classe dos ovos ou das larvas. Vamos supor que exista competição entre os indivíduos neste estágio, ou seja, vamos supor que a taxa de sobrevivência neste período é dada por uma certa função $g$ dependente da densidade, uma função decrescente, $\operatorname{com} g(0)=1$ e $\lim _{x \rightarrow \infty} g(x)=0$. Vários exemplos para a 
escolha da função $g$ são dados em [7]. Assim, a densidade na classe etária 1 é dada por

$$
x_{t}^{1}=g\left(w_{t}\right) w_{t}=g\left(w_{t}\right) \sum_{i=1}^{N} f_{i} x_{t}^{i},
$$

e o sistema dado por (2.1) torna-se

$$
\begin{aligned}
x_{t+1}^{1} & =g\left(w_{t}\right) \sum_{i=1}^{N} f_{i} x_{t}^{i}, \\
x_{t+1}^{i+1} & =p_{i} x_{t}^{i}, \quad i=2, \ldots, N-1, \\
w_{t} & =\sum_{i=1}^{N} f_{i} x_{t}^{i} .
\end{aligned}
$$

Substituindo as expressões para $x_{t}^{i}$ recursivamente na expressão para $w_{t}$, temos

$$
w_{t}=R_{0} \sum_{i=1}^{N} m_{i} w_{t-i} g\left(w_{t-i}\right) .
$$

Fazendo $z_{t}^{1}=g\left(w_{t-1}\right) w_{t-1}$ e $z_{t}^{i}=z_{t-1}^{i-1}, \quad i=2, \ldots, N-1$ obtemos o sistema

$$
\begin{aligned}
w_{t+1} & =R_{0}\left(m_{1} w_{t} g\left(w_{t}\right)+\sum_{i=2}^{N} m_{i} z_{t}^{i-1}\right) \\
z_{t+1}^{1} & =g\left(w_{t}\right) w_{t}, \\
z_{t+1}^{2} & =z_{t}^{1} \\
& \vdots \\
z_{t+1}^{N-1} & =z_{t}^{N-2} .
\end{aligned}
$$

\section{Pontos de Equilíbrio}

É fácil verificar que o sistema (2.5) possui dois pontos de equilíbrio: o ponto de equilíbrio trivial e um ponto de equilíbrio positivo, que satisfaz

$$
g\left(w^{*}\right)=\frac{1}{R_{0}} .
$$

Como $g(x) \leq 1$ temos que ter $R_{0} \geq 1$ para que a equação (2.6) possa ser satisfeita. Se $R_{0}=1$ temos $g\left(w^{*}\right)=1 \Rightarrow w^{*}=0$. Portanto a condição para a existência de um equilíbrio homogêneo não trivial é

$$
R_{0}>1
$$

\section{Equação Característica}

A matriz jacobiana no equilíbrio não trivial é dada por

$$
J\left(\mathbf{x}^{*}\right)=\left[\begin{array}{ccccc}
f_{1} h^{\prime}\left(w^{*}\right) & f_{2} h^{\prime}\left(w^{*}\right) & \cdots & f_{N-1} h^{\prime}\left(w^{*}\right) & f_{N} h^{\prime}\left(w^{*}\right) \\
p_{1} & p_{2} & & \\
& & \ddots & \\
& & & p_{N-1} &
\end{array}\right],
$$


onde $h(x)=g(x) x$. Utilizando a relação

$$
J\left(\mathbf{x}^{*}\right) \mathbf{v}=\lambda \mathbf{v}
$$

onde $\mathbf{v}=\left(v_{1}, v_{2}, \ldots, v_{N}\right)^{T}$ é o autovetor associado com o autovalor $\lambda$ de $J\left(\mathbf{x}^{*}\right)$, obtemos a seguinte equação característica para os autovalores não-nulos de $J\left(\mathbf{x}^{*}\right)$

$$
\sum_{k=1}^{N} \frac{m_{k}}{\lambda^{k}}=\frac{1}{R_{0} h^{\prime}\left(w^{*}\right)}=\frac{1}{1-H}
$$

onde $H=\frac{-w^{*} g\left(w^{*}\right)}{g^{\prime}\left(w^{*}\right)}$.

\section{Modelo Acoplado}

Nesta seção, vamos considerar uma rede com $n$ sítios, tal que em cada sítio exista uma população estruturada em $N$ classes etárias, onde os indivíduos de cada classe etária migram para os dois sítios mais próximos com uma taxa de migração específica para sua classe. Após este processo de migração, ocorrem os processos de reprodução e sobrevivência. Matematicamente, o modelo pode ser expresso por

$$
\begin{aligned}
x_{t+1, k}^{1}= & g\left(w_{t, k}\right) w_{t, k}, \\
x_{t+1, k}^{2}= & \left(1-\mu_{1}\right) p_{1} x_{t, k}^{1}+\frac{\mu_{1}}{2} p_{1}\left(x_{t, k-1}^{1}+x_{t, k+1}^{1}\right), \\
\vdots & \\
x_{t+1, k}^{N}= & \left(1-\mu_{N-1}\right) p_{N-1} x_{t, k}^{N-1}+\frac{\mu_{N-1}}{2} p_{N-1}\left(x_{t, k-1}^{N-1}+x_{t, k+1}^{N-1}\right), \\
w_{t, k}= & f_{1}\left[\left(1-\mu_{1}\right) x_{t, k}^{1}+\frac{\mu_{1}}{2}\left(x_{t, k-1}^{1}+x_{t, k+1}^{1}\right)\right] \\
& +f_{2}\left[\left(1-\mu_{2}\right) x_{t, k}^{2}+\frac{\mu_{2}}{2}\left(x_{t, k-1}^{2}+x_{t, k+1}^{2}\right)\right] \\
& +\cdots+f_{N}\left[\left(1-\mu_{N}\right) x_{t, k}^{N}+\frac{\mu_{N}}{2}\left(x_{t, k-1}^{N}+x_{t, k+1}^{N}\right)\right],
\end{aligned}
$$

onde $\mu_{i}$ são as taxas de migração específicas para cada classe etária $i, i=1,2, \ldots, N$. Utilizando as variáveis

$$
\begin{gathered}
z_{t+1, k}^{1}=R_{0}\left(\left(1-\mu_{1}\right) h\left(w_{t, k}\right)+\frac{\mu_{1}}{2}\left[h\left(w_{t, k-1}\right)+h\left(w_{t, k+1}\right)\right]\right), \\
z_{t+1, k}^{i}=\left(\left(1-\mu_{i}\right) z_{t, k}^{i-1}+\frac{\mu_{i}}{2}\left[z_{t, k-1}^{i-1}+z_{t, k+1}^{i-1}\right]\right), i=2, \ldots, N-1,
\end{gathered}
$$


obtemos o sistema

$$
\begin{aligned}
w_{t+1, k}= & R_{0} m_{1}\left[\left(1-\mu_{1}\right) h\left(w_{t, k}\right)+\frac{\mu_{1}}{2}\left(h\left(w_{t, k-1}\right)+h\left(w_{t, k+1}\right)\right)\right] \\
& +m_{2}\left[\left(1-\mu_{2}\right) z_{t, k}^{1}+\frac{\mu_{1}}{2}\left(z_{t, k-1}^{1}+z_{t, k+1}^{1}\right)\right] \\
& +\cdots+m_{N}\left[\left(1-\mu_{N}\right) z_{t, k}^{N-1}+\frac{\mu_{N}}{2}\left(z_{t, k-1}^{N-1}+z_{t, k+1}^{N-1}\right)\right] \\
z_{t+1, k}^{1}= & R_{0}\left(\left(1-\mu_{1}\right) h\left(w_{t, k}\right)+\frac{\mu_{1}}{2}\left[h\left(w_{t, k-1}\right)+h\left(w_{t, k+1}\right)\right]\right) \\
z_{t+1, k}^{i}= & \left(\left(1-\mu_{i}\right) z_{t, k}^{i-1}+\frac{\mu_{i}}{2}\left[z_{t, k-1}^{i-1}+z_{t, k+1}^{i-1}\right]\right), i=2, \ldots, N-1 .
\end{aligned}
$$

Note que, fazendo $\mu_{1}=\mu_{2}=\cdots=\mu_{N}=0$ em (3.1), obtemos o sistema (2.5). Portanto, o sistema acima é de fato uma extensão de (2.5) ao caso de uma rede com $n$ patches. O sistema (3.1) é válido para cada sítio $k, k=1,2, \ldots, n$. Utilizamos condições de fronteira periódicas, desta forma a rede pode ser vista como um anel onde o sítio 1 e o sítio $n$ estão interligados. Isto é feito afim de eliminar possíveis efeitos de fronteira na dinâmica do sistema. Dada esta imposição geométrica, os valores do índice $k$ em (3.1) devem ser interpretados com cuidado: $k=n+1$ deve ser interpretado como $k=1$, assim como $k=0$ significa $k=n$. Esta observação é especialmente importante para a obtenção da matriz jacobiana no ponto de equilíbrio positivo, que é determinado a seguir.

\section{Equilíbrio homogêneo positivo}

É fácil verificar que o sistema (3.1) possui um único ponto de equilíbrio homogêneo positivo $W^{*}=\left(w^{*}, \ldots, w^{*}\right)$, onde $w^{*}$ satisfaz

$$
g\left(w^{*}\right)=\frac{1}{R_{0}}
$$

Assim, a condição para a existência de um equilíbrio homogêneo positivo é $R_{0}>1$, como no caso desacoplado.

\section{A estabilidade do ponto de equilíbrio homogêneo positivo}

Para proceder à análise de estabilidade do ponto de equilíbrio homogêneo positivo, calculamos a matriz jacobiana no equilíbrio $\left(w^{*}, w^{*}, \ldots, w^{*}\right)^{T}$,

$$
J\left(W^{*}\right)=\left[\begin{array}{ccccc}
A_{1} & A_{2} & \cdots & A_{N-1} & A_{N} \\
B_{1} & 0 & \cdots & 0 & 0 \\
0 & B_{2} & 0 & & 0 \\
\vdots & \ddots & \ddots & \ddots & \vdots \\
0 & \cdots & 0 & B_{N-1} & 0
\end{array}\right]
$$


Os blocos $A_{i}, i=1, \ldots, N$ e $B_{l}, l=1, \ldots, N-1$ são matrizes circulantes $n \times n$ dadas por

$$
A_{i}=\left[\begin{array}{ccccc}
\alpha_{i} & \beta_{i} & & & \beta_{i} \\
\beta_{i} & \alpha_{i} & \beta_{i} & & \\
& \ddots & \ddots & \ddots & \\
& & \beta_{i} & \alpha_{i} & \beta_{i} \\
\beta_{i} & & & \beta_{i} & \alpha_{i}
\end{array}\right] \text { e } B_{l}=\left[\begin{array}{ccccc}
\gamma_{l} & \xi_{l} & & & \xi_{l} \\
\xi_{l} & \gamma_{l} & \xi_{l} & & \\
& \ddots & \ddots & \ddots & \\
& & \xi_{l} & \gamma_{l} & \xi_{l} \\
\xi_{l} & & & \xi_{l} & \gamma_{l}
\end{array}\right]
$$

onde

$$
\begin{array}{rlrl}
\alpha_{1} & =\left(1-\mu_{1}\right) m_{1}(1-H), & & \\
\beta_{1}=\frac{\mu_{1}}{2} m_{1}(1-H), & & \\
\alpha_{i}=m_{i}\left(1-\mu_{i}\right), & & i=2, \ldots, N, \\
\beta_{i} & =m_{i} \frac{\mu_{i}}{2}, & & i=2, \ldots, N, \\
\gamma_{1} & =\left(1-\mu_{1}\right)(1-H), & & \\
\xi_{1}=\frac{\mu_{1}}{2}(1-H), & & \\
\gamma_{i}=\left(1-\mu_{i}\right), & & i=2, \ldots, N-1, \\
\xi_{i} & =\frac{\mu_{i}}{2}, & &
\end{array}
$$

e $1-H=R_{0} h^{\prime}\left(w^{*}\right)$. Dada a forma da matriz $J\left(W^{*}\right)$, temos que se $0<H<2 W^{*}$ é assintoticamente estável, como demonstra o resultado a seguir.

Teorema 3.1. Se $0<H<2$ então o equilíbrio $W^{*}=\left(w^{*}, w^{*}, \ldots, w^{*}\right)^{T}$ é assintoticamente estável.

Demonstração. Pelo Teorema de Gershgorin[4] o espectro de $J$ está contido na união das bolas $S_{i}\left(c_{i}, \phi_{i}\right)$, onde $c_{i}=J_{i i}$ é o centro e $\phi_{i}=\sum_{i=1}^{n N}\left|J_{i j}\right|$ é o raio das bolas $S_{i}$. Assim, se

$$
\begin{aligned}
& \left|\alpha_{1}\right|+\phi_{i}<1, \quad 1 \quad \leq i \leq n, \\
& \phi_{i}<1, \quad n+1 \leq i \leq N n,
\end{aligned}
$$

a estabilidade do sistema está garantida. Lembrando que, por hipótese, $0<H<2$, temos

$$
\begin{aligned}
\left|\alpha_{1}\right|+\quad \phi_{i} & =\left|\alpha_{1}\right|+2\left|\beta_{1}\right|+\sum_{j=2}^{N-1} \alpha_{j}+2 \beta_{j} & \\
& =m_{1}|1-H|+\sum_{j=2}^{N} m_{j}<\sum_{j=1}^{N} m_{j}=1, & 1 \leq i \leq n \\
\phi_{i} & =\left|\gamma_{1}\right|+2\left|\xi_{1}\right|=|1-H|<1, & n+1 \leq i \leq 2 n \\
\phi_{i} & =\gamma_{j}+2 \xi_{j}=\left(1-\mu_{2}\right)+2 \frac{\mu_{j}}{2}=1, & j n+1 \leq i \leq(j+1) n
\end{aligned}
$$

o que completa o resultado.

Com um pouco de álgebra [1], temos que os $n N$ autovalores $\sigma$ da matriz jacobiana $J\left(W^{*}\right)$ são as raízes de

$$
\frac{\lambda_{j}^{1}}{\sigma}+\sum_{l=2}^{N} \frac{\lambda_{j}^{l} \prod_{k=1}^{l-1} \nu_{j}^{k}}{\sigma^{l}}=1, \quad j=0, \ldots, n-1,
$$


onde

$$
\lambda_{j}^{l}=\alpha_{l}+2 \beta_{l} \cos \frac{2 \pi j}{n}, \quad j=0, \ldots, n-1,
$$

são os $n$ autovalores de $A_{l}$ e

$$
\nu_{j}^{k}=\gamma_{k}+2 \xi_{k} \cos \frac{2 \pi j}{n}, \quad j=0, \ldots, n-1,
$$

são os $n$ autovalores de $B_{k}$. Da equação (3.6) obtemos que o polinômio característico é o produto dos polinômios

$$
p_{j}(\sigma)=\sigma^{N}-\lambda_{j}^{1} \sigma^{N-1}-\sum_{l=2}^{N} \lambda_{j}^{l} \sigma^{N-l} \prod_{k=1}^{l-1} \nu_{j}^{k}, \quad j=0, \ldots, N-1 .
$$

Observando a forma de $p_{0}$, temos que a migração não pode vir a estabilizar um sistema de populações locais instáveis, como demonstra o resultado a seguir.

Teorema 3.2. Todo autovalor do modelo local é autovalor do sistema acoplado. Desse modo, o movimento migratório entre patches não pode vir a estabilizar um sistema desacoplado instável.

Demonstração. Tomando $j=0$ na equação característica para o modelo acoplado temos

$$
\frac{\alpha_{1}+2 \beta_{1}}{\sigma}+\sum_{l=2}^{N} \frac{\alpha_{l}+2 \beta_{l} \prod_{k=1}^{l-1} \gamma_{k}+2 \xi_{k}}{\sigma^{l}}=1 .
$$

Substituindo as expressões para $\alpha_{l}, \beta_{l}, \gamma_{k}, \xi_{k}$ obtemos

$$
\sum_{l=1}^{N} \frac{m_{l}}{\sigma^{l}}=\frac{1}{R_{0} h^{\prime}(w *)}=\frac{1}{1-H}
$$

que é a equação característica para o modelo local (2.10). Assim, temos que os autovalores do modelo local satisfazem a equação característica para o modelo acoplado, completando a demonstração.

Em certos casos, a migração não altera a estabilidade de $W^{*}$. Entre estes, temos o caso em que o processo migratório independe da estrutura etária da população, como é demonstrado no teorema a seguir.

Teorema 3.3. Se $\mu_{i}=c, i=1, \ldots, N$, o movimento migratório não influencia na estabilidade de $W^{*}$.

Demonstração. Neste caso, a equação característica do modelo acoplado torna-se

$$
(1-H) \sum_{i=1}^{N} m_{i}\left(\frac{(1-c)+c \cos \frac{2 \pi j}{n}}{\sigma}\right)^{i}=1 \text {. }
$$


Portanto, se $\lambda$ satisfaz a equação característica (2.10) do modelo local, então

$$
\sigma=\lambda\left((1-c)+c \cos \frac{2 \pi j}{n}\right)
$$

satisfaz à equação característica para o modelo acoplado descrita acima, para $j=$ $0, \ldots, n-1$. Como $\left|\left((1-c)+c \cos \frac{2 \pi j}{n}\right)\right|<1$, temos $|\sigma| \leq|\lambda|$, ocorrendo a igualdade (em módulo e em sinal) quando $j=0$.

\section{A Instabilidade Causada pelo Movimento Migratório}

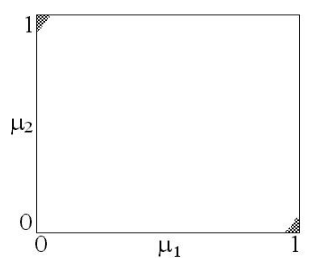

(a) $m_{1}=0.35 \quad H=2.1$

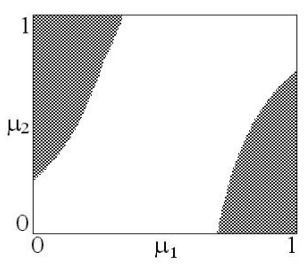

(d) $m_{1}=0.6 \quad H=3.4$

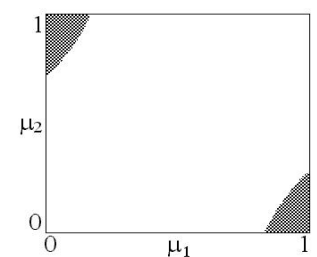

(b) $m_{1}=0.4 \quad H=2.5$

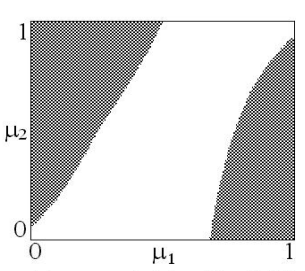

(e) $m_{1}=0.66 \quad H=3.75$

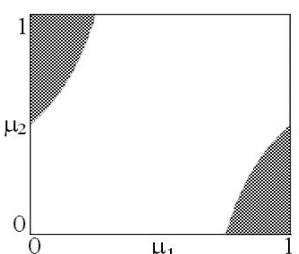

(c) $m_{1}=0.5 \quad H=2.99$

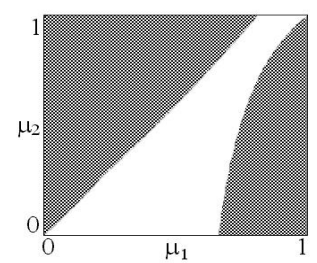

(f) $m_{1}=0.666 H=3.99$

Figura 1: Teste de Jury aplicado a todas as possíveis configurações para as taxas migratórias em uma rede com 20 sítios com populações locais estruturadas em duas classes etárias.

Por outro lado, a migração pode, de fato, vir a desestabilizar uma rede de populações desacopladas estáveis se as taxas migratórias forem fortemente relacionadas com a idade dos indivíduos. Para isto, vamos considerar um caso específico: uma metapopulação formada por 20 sítios, tal que em cada sítio existe uma subpopulação estruturada em duas classes etárias e aplicar o teste de Jury[3] ao polinômio característico do sistema acoplado. O teste de Jury fornece condições necessárias e suficientes sobre os coeficientes de um polinômio para que suas raízes estejam dentro do disco unitário, fornecendo portanto a região de estabilidade do sistema. Na figura 1, escolhemos parâmetros da dinâmica local $\left(m_{1}\right.$ e $\left.H\right)$ tais que o ponto de equilíbrio $W^{*}$ é assintoticamente estável na ausência de migração e variamos as taxas migratórias $\mu_{1}$ e $\mu_{2}$. Para visualizar os resultados, pintamos cada ponto $\left(\mu_{1}, \mu_{2}\right)$ do quadrado unitário de acordo com o resultado do teste: branco representa estabilidade, cinza representa instabilidade. Assim, as regiões hachuradas representam instabilidades causadas somente pela dispersão. Nos testes realizados, observamos 

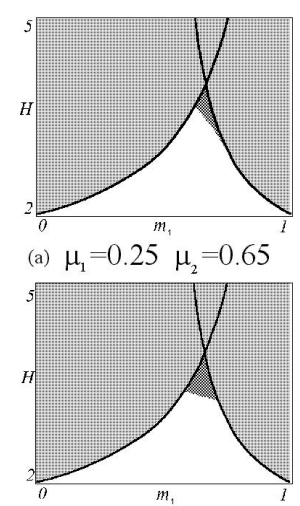

(d) $\mu_{1}=0.75 \mu_{2}=0.2$

$\square$ Sistema acoplado estável
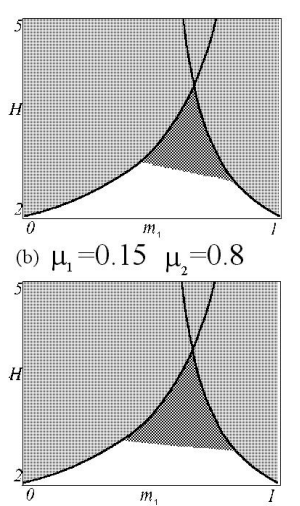

(e) $\mu_{1}=0.85 \mu_{2}=0.1$
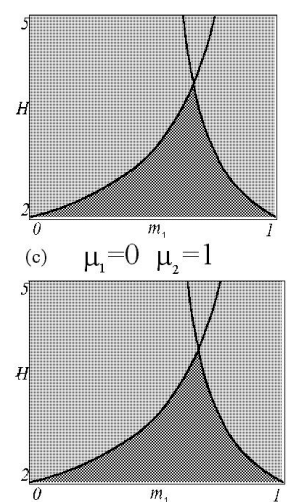

(f) $\quad \mu_{1}=1 \quad \mu_{2}=0$

瞒 Sistema desacoplado instável In: Instabilidade gerada pela migração

Figura 2: Teste de Jury aplicado a diversas configurações para uma metapopulação com duas classes etárias e 20 sítios. As curvas em preto definem a região de estabilidade para o modelo local, delimitada por $H=0, H=1+\frac{1}{1-m_{1}} \mathrm{e}$ $H=1+\frac{1}{2 m_{1}-1}$.

que, à medida que $H$ cresce, a região de instabilidade do modelo acoplado aumenta. Para $H \approx 4$, temos a maior região de instabilidade causada pela migração, como pode ser observado na Figura 1. Na Figura 2, temos a região de estabilidade para possíveis configurações no modelo acoplado, obtida através da aplicação do teste de Jury para diferentes valores de $\mu_{1}$ e $\mu_{2}$. Nesta figura, diferenciamos a região onde o sistema desacoplado é instável, para uma fácil identificação da região de instabilidade gerada apenas pela migração. As Figuras 2(a), 2(b) e 2(c) mostram que a região de estabilidade é reduzida gradualmente a região limite $0<H<2$ à medida que nos aproximamos de $\mu_{1}=1$ e $\mu_{2}=0$. O mesmo ocorre ao nos aproximarmos de $\mu_{1}=0$ e $\mu_{2}=1$, como indicam as Figuras 2(d), 2(e) e 2(f). Na verdade, isto ocorre independentemente do número de classes considerado: para uma rede tal que $\mu_{1}=1$ e $\mu_{i}=0$ se $i \neq 1, W^{*}$, temos

$$
p_{j}(\sigma)=\sigma^{N}-\left(m_{1} \sigma^{N-1}+\cdots+m_{N-1} \sigma+m_{N}\right)(1-H) \cos \frac{2 \pi j}{n},
$$

portanto

$$
p_{j}(1)=1-(1-H) \cos \frac{2 \pi j}{n} .
$$

Isto implica que, se $n$ é par, $p_{\frac{n}{2}}(1)>0 \Leftrightarrow H<2$. Como $p_{j}$ deve satisfazer $p_{j}(1)>0$ para todo $j$ para que os autovalores do sistema sejam menores que um em módulo, temos que se $H>2$ o sistema (sob as configurações citadas) apresenta instabilidade. Este caso representa a situação onde apenas os jovens podem migrar. Para o caso contrário, onde todos os indivíduos migram e apenas os mais jovens permanecem 
em seu sítio de origem, o mesmo ocorre. Neste caso, temos $\mu_{1}=0$ e $\mu_{i}=1$, $i=2, \ldots, N, W^{*}$ e os polinômios $p_{j}(\sigma)$ são dados por

$$
p_{j}(\sigma)=\sigma^{N}-(1-H)\left(m_{1} \sigma^{N-1}+\sum_{i=2}^{N} m_{i} \sigma^{N-i}\left(\cos \frac{2 \pi j}{n}\right)^{i-1}\right) .
$$

Assim,

$(-1)^{N} p_{j}(-1)=1-(-1)^{N}(1-H)\left(m_{1}(-1)^{N-1}+\sum_{i=2}^{N} m_{i}(-1)^{N-i}\left(\cos \frac{2 \pi j}{n}\right)^{i-1}\right)$.

Assumindo $n$ par temos que $(-1)^{N} p_{\frac{n}{2}}(-1)=2-H$ e, portanto, $(-1)^{N} p_{\frac{n}{2}}(-1)>$ $0 \Leftrightarrow H<2$. Como, pelo teste de Jury, $(-1)^{N} p_{j}(-1)>0$ é condição necessária para que não ocorram autovalores negativos com módulo maior que um, temos que o sistema sob as configurações citadas é estável se, e somente se, $H<2$. Assim, temos que a condição para estabilidade $0<H<2$ dada pelo Teorema 3.1 não pode ser melhorada sem um conhecimento maior sobre o mecanismo de migração e a relação deste com a estrutura etária da população.

Abstract In this work we obtain a discrete model for a metapopulation of a single species with overlapping generations. We show that a migration mechanism which depends only on age can not stabilize a previously unstable homogeneous equilibrium, but can drive a stable uncoupled system to instability, which characterizes migration as a destabilizing mechanism.

\section{Referências}

[1] M.L. Castro, "O Efeito da Migração sobre a Homogeneidade em Metapopulações", Dissertação de Mestrado, Universidade Federal do Rio Grande do Sul, 2001.

[2] A. Hastings, Age dependent dispersal is not a simple process: density dependence, stability, and chaos, Theor. Pop. Biol., 41 (1992), 388-400.

[3] E.I. Jury, The inners approach to some problem of system theory, IEEE Trans. Automatic Contr., 16 (1971), 233-240.

[4] P. Lancaster and M. Tismenetsky, "The Theory of Matrices", Academic Press, London, 1985.

[5] P. Rohani, R.M. May and M.P. Hassel, Metapopulation and equilibrium stability: the effects of spatial structure, J. Theor. Biol., 181 (1996), 97-109.

[6] J.A.L. Silva, M.L. Castro and D.A.R. Justo, Stability in a density-dependent metapopulation model, Bull. Math. Biol., to appear.

[7] J.A.L. Silva and T.G. Hallam, Compensation and stability in nonlinear matrix models, Mathematical Biosciences, 110 (1992), 67-101. 